[19] H. G. Zhu, J. Ji, Q. G. Tan, M. A. Barbosa, J. C. Shen, Biomacromolecules 2003, 4, 378 .

[20] X. Jiang, P. T. Hammond, Langmuir 2000, 16, 8501.

[21] X. Jiang, S. L. Clark, P. T. Hammond, Adv. Mater. 2001, 13, 1669.

[22] M. Gao, J. Sun, E. Dulkeith, N. Gaponik, U. Lemmer, J. Feldmann, Langmuir 2002, 18, 4098.

[23] H. Jang, S. Kim, K. Char, Langmuir 2003, 19, 3094.

[24] S. Granick, S. A. Sukhishvili, J. Am. Chem. Soc. 2000, 122, 9550.

[25] S. Y. Yang, M. F. Rubner, J. Am. Chem. Soc. 2002, 124, 2100.

[26] J. J. Harris, P. M. DeRose, M. L. Bruening, J. Am. Chem. Soc. 1999, 121, 1978.

[27] F. Shi, B. Dong, D. L. Qiu, J. Q. Sun, T. Wu, X. Zhang, Adv. Mater. 2002, 14,805

[28] Y. L. Loo, R. L. Willett, K. W. Baldwin, J. A. Rogers, J. Am. Chem. Soc. 2002, 124, 7654.

[29] J. Zaumseil, M. A. Meitl, J. W. P. Hsu, B. R. Acharya, K. W. Baldwin, Y. L. Loo, J. A. Rogers, Nano Lett. 2003, 3, 1223.

[30] M. C. Berg, J. Choi, P. T. Hammond, M. F. Rubner, Langmuir 2003, $19,2231$.

[31] The adsorption behavior of PAH on a hydrophobic surface and effects of pattern features on the process will be theoretically and experimentally characterized in two separate papers.

[32] C. Poncet, F. Tiberg, R. Audebert, Langmuir 1998, 14, 1697.

[33] A. V. Dobrynin, M. Rubinstein, Macromolecules 2002, 35, 2754.

[34] S. L. Clark, M. F. Montague, P. T. Hammond, Macromolecules 1997, $30,7237$.

[35] M. Fang, C. H. Kim, G. B. Saupe, H.-N. Kim, C. C. Waraksa, T. Miwa, A. Fujishima, T. E. Mallouk, Chem. Mater. 1999, 11, 1526.

[36] J. Suh, H.-J. Paik, B. K. Hwang, Bioorg. Chem. 1994, 22, 318.

[37] Y. Yoshikawa, H. Matsuoka, N. Ise, Br. Polym. J. 1986, 18, 242

[38] K. Ariga, Y. Lvov, T. Kunitake, J. Am. Chem. Soc. 1997, 119, 2224

[39] A. D. Child, J. R. Reynolds, Macromolecules 1994, 27, 1975.

[40] M. J. Owen, P. J. Smith, J. Adhesion Sci. Technol. 1994, 8, 1063.

\section{Room-Temperature Nanoimprint Lithography of Non-thermoplastic Organic Films}

\author{
By Dario Pisignano,* Luana Persano, \\ Maria Filomena Raganato, Paolo Visconti, \\ Roberto Cingolani, Giovanna Barbarella, \\ Laura Favaretto, and Giuseppe Gigli
}

Nanoimprint lithography (NIL) ${ }^{[1]}$ enables the transfer of master patterns fabricated by conventional lithographic tech-

[*] Dr. D. Pisignano, L. Persano, M. F. Raganato, Dr. P. Visconti,

Prof. R. Cingolani, Dr. G. Gigli

National Nanotechnology Laboratory (NNL)

Istituto Nazionale di Fisica della Materia (INFM)

c/o Dipartimento di Ingegneria dell'Innovazione

via Arnesano, 1-73100 Lecce (Italy)

E-mail: dario.pisignano@unile.it

Dr. D. Pisignano

Dipartimento di Fisica, Università di Lecce

via Arnesano, I-73100 Lecce (Italy)

Dr. G. Barbarella, L. Favaretto

Consiglio Nazionale delle Ricerche (CNR), ISOF

Area della Ricerca di Bologna

via Gobetti 101, I-40129 Bologna (Italy) niques with very high fidelity by exploiting the glass transition of polymers. NIL uses a hard ${ }^{[1]}$ mold to cheaply imprint micro- and nanostructures onto large areas of the target compounds, without exposure to radiation, development, or etching processes. This technique has enabled the production of sub-100 nm structures ${ }^{[2]}$ on 6 in. $(1$ in. $=2.54 \mathrm{~cm})$ wafers, ${ }^{[3]}$ nanoscale field-effect transistors, ${ }^{[4]}$ metal-semiconductormetal photodetectors ${ }^{[5]}$ and three-dimensional patterns. ${ }^{[6]}$

During the imprinting, the mold is positioned onto a cast film of the target polymer, and then the system is heated at a temperature considerably higher than the glass transition temperature, $T_{\mathrm{g}}$, of the target. At high temperature, the target material takes the shape of the master due to the its low viscosity. Finally, the system is cooled to below $T_{\mathrm{g}}$, thus freezing a negative copy of the master pattern into the target. In order to obtain a high-quality pattern transfer by this thermal cycle, good thermoplastic behavior of the target compounds is needed. Unfortunately, this is difficult to accomplish with small molecules, thus preventing their use as directly printed active materials, despite the fact that many low molar mass compounds have been found to exhibit excellent luminescence and optical gain performances. ${ }^{[7]}$ Clearly, the development of alternative lithography methods that allow the patterning of these materials would allow them to be employed for one-step realization of functional optoelectronic devices.

Recently, room-temperature (RT) embossing ${ }^{[8]}$ has been proposed as an alternative to conventional NIL. RT-NIL offers some advantages over hot nanoimprinting, such as the possibility to perform multiple imprinting processes on the same resist layer.

However, this technique was reported to exploit the plastic flow of thermoplastic polymers, such as polystyrene, which can also be printed by conventional, high-temperature NIL. For active low-molar-mass organic semiconductors with poor thermoplastic behavior, room-temperature imprinting could be the only process that permits the transfer of a height profile into films of this very important class of molecules. In this work, we applied RT-NIL to a number of such compounds, and carefully investigated the changes in the optical properties (absorbance, luminescence, and quantum yield), thus ruling out any possible degradation of the active materials after patterning.

Our lithography process is schematized in Figure 1. Master patterns of period, $\Lambda$, between 400 and $600 \mathrm{~nm}$, and total area $3 \mathrm{~mm} \times 8 \mathrm{~mm}$, were fabricated on silicon by electron-beam lithography (Leica LION LVI system working at an acceleration energy of $2.5 \mathrm{keV})$, lift-off, and reactive ion etching $\left(\mathrm{CF}_{4} /\right.$ Ar mixture chosen in order to minimize the plasma-induced polymer deposition). The low-molar-mass compounds, whose molecular structures are displayed in Figure 2, include a rigidcore oligothiophene (1) ${ }^{[9]}$ a diamine derivative $\left(N, N^{\prime}\right.$-diphenyl- $N, N^{\prime}$-bis(3-methylphenyl)-1,1'-biphenyl-4,4'diamine) (2), different linear oligothiophene- $S, S$-dioxides $(\mathbf{3 , 5}, 7),{ }^{[10,11]}$ a phenyl-based oligomer with one central thiophene moiety (4) ${ }^{[11]}$ and a molecule with a branched benzo[b]thiophene based structure (6). The organic films were cast onto Corning 

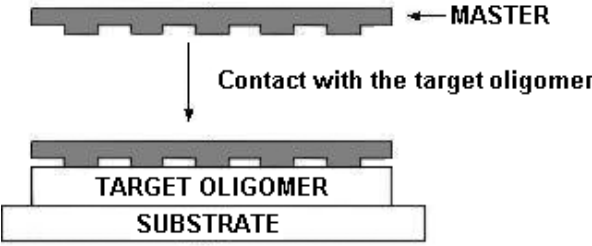

Applying pressure (No heating)

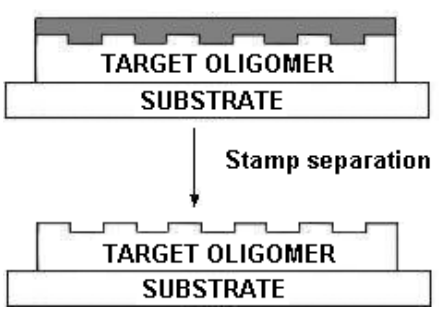

Figure 1. Schematic diagram of the process of room temperature nanoimprint lithography (features not to scale).

glass substrates, from chloroform and dichloromethane solutions, at a thickness between 200 and $500 \mathrm{~nm}$ (spin-coating speed $2000 \mathrm{rpm}$ ). The master, whose cross-section is displayed in Figure 3, was then used as a mold for nanoimprint, employing a precision manual press (PW100 P/O/Weber, Germany) at room temperature, in air, with an applied force in the range $3-5 \mathrm{kN}$. No antisticking layer was employed in the imprinting process. The absorption (Abs) and photoluminescence (PL) spectra of the compounds before and after patterning, were measured by a UV-visible spectrophotometer (Varian Cary $100-$ Scan) and by exciting samples with a He-Cd laser $(\lambda=325 \mathrm{~nm})$. The external PL efficiency, $\eta_{\mathrm{PL}}$, was determined

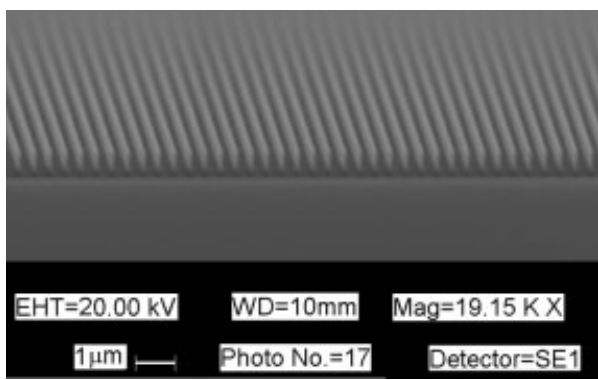

Figure 3. Cross-sectional view of a $400 \mathrm{~nm}$ period master grating imaged by scanning electron microscopy. The features are more than $500 \mathrm{~nm}$ high (aspect ratio larger than 2.5 ).
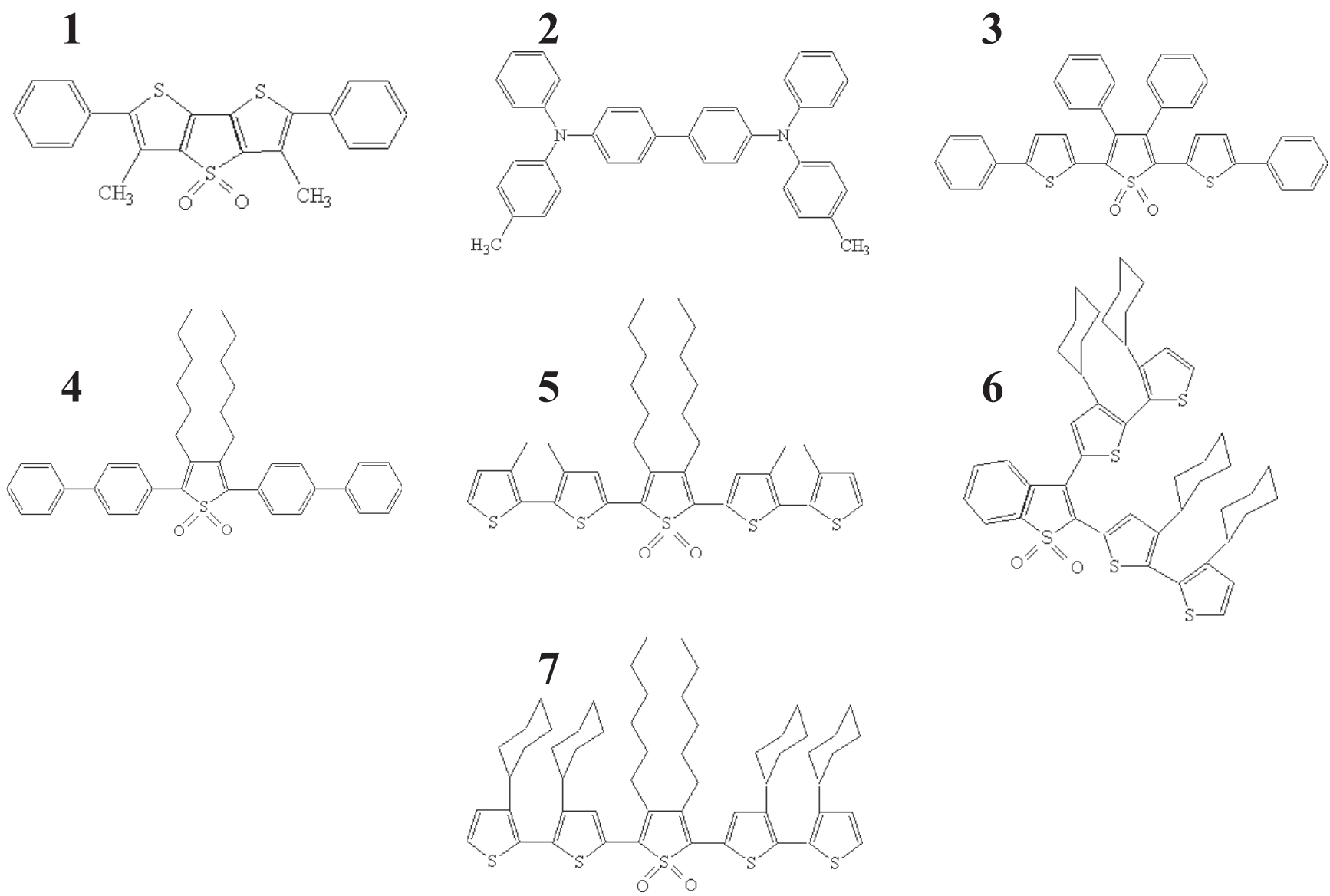

Figure 2. Molecular structures of the imprinted compounds. Compound 2 was purchased from Sigma-Aldrich and used as received. Details of the synthesis procedures of the thiophene-based compounds (1 and $3-7$ ) are reported elsewhere $[9,11]$. 
by an integrating sphere. The optical measurements were carried out under nitrogen flux to inhibit photo-oxidation.

Prior to the RT-NIL experiments, we carried out extensive studies (particularly on 7), aiming at obtaining patterns either by high-temperature NIL or by soft molding, which employs elastomeric replicas of the master placed in conformal contact with the target. ${ }^{[12]}$ We observed a strong sticking of the heated oligomer to the molds without achieving surface patterning, due to the lack of temperature-induced plastic deformations and to the weak adhesion of the fluid oligomer to the substrate. Instead, the plan-view and the cross-section of a $400 \mathrm{~nm}$ period oligomer grating printed by RT-NIL, imaged by atomic force microscopy (AFM; Fig. 4), show a well-defined pattern, the resolution achieved being dependent on that of the starting masters.

The behavior of the oligomers under an applied stress can be understood by considering the low-molar-mass limit of the polymers. In general, the mechanical response of amorphous materials to an external stress is ruled by their visco-elastic behavior, such as the initial elastic response and the viscous response to external stress. In particular, local motions are due to conformational energy barriers, mainly energy-activated processes, whereas long-range motions, namely the plastic flow of the materials, occur upon weakening the intermolecular entanglements. ${ }^{[13]}$ These two processes qualitatively

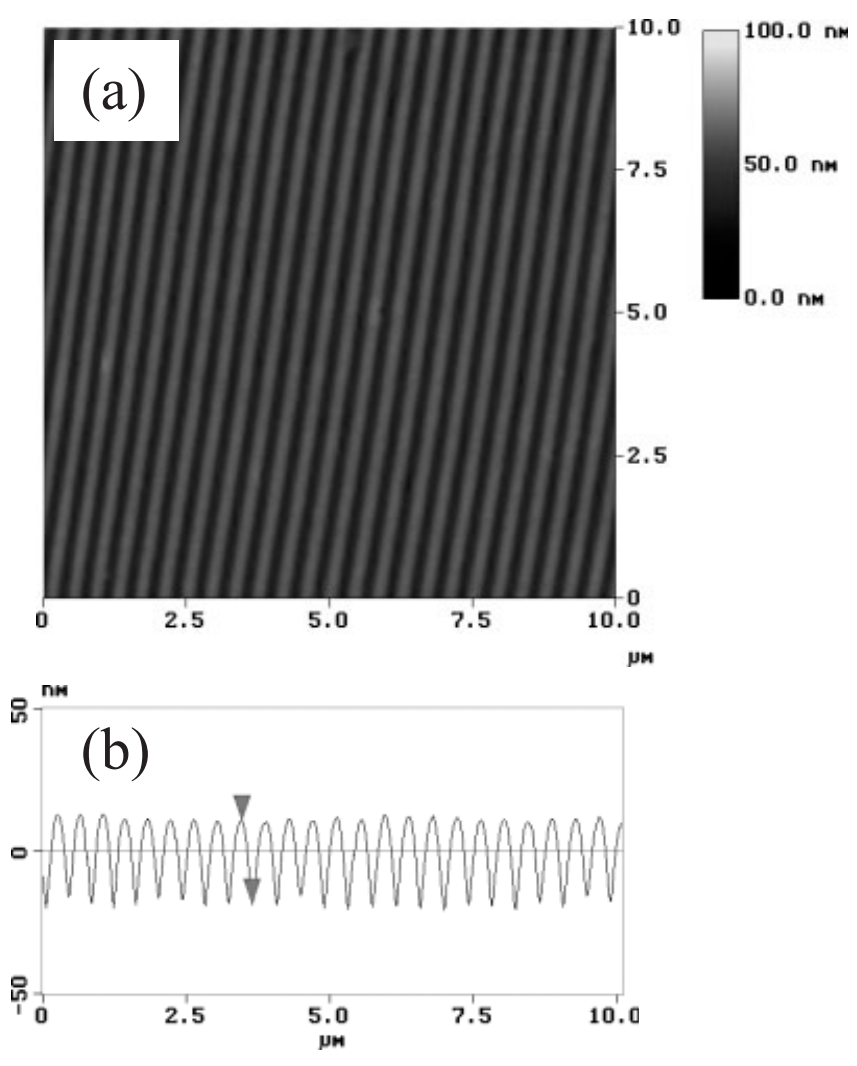

Figure 4. a) AFM two-dimensional view of a $400 \mathrm{~nm}$ period grating transferred into a low-molar-mass, non-thermoplastic organic semiconductor (7), and corresponding cross-section (b). The height of the features is about $30 \mathrm{~nm}$. correspond to the secondary $(\beta-)$ and to the main or structural $(\alpha-)$ relaxations, respectively, which are observable in amorphous solids and fluids. ${ }^{[14]}$ With increasing temperature in conventional NIL, a polymer first reaches a rubber-elastic region, preserving its network of entanglements. This leads to reversible shape changes, and to a weak, plateau-like dependence of the shear compliance, $J$, on the flow time, $t .^{[15]}$ One is then able to drive the material to its terminal flow region, in which the pressure-induced deformations become irreversible. This is characterized by a linear relation, $J$ proportional to $t$, and is due to the viscous molecular flow slipping out of the chain entanglements. Interestingly, the rubber-elastic behavior is more evident in polymers heavier than a material-dependent critical mass, $M_{\mathrm{C}}\left(\sim 10^{3} \mathrm{~g} \mathrm{~mol}^{-1}\right.$ for poly(methyl methacrylate $)^{[15]}$, due to the enhanced entanglements, whereas lighter compounds can easily reach the terminal flow region. This means that irreversible deformations can be induced more easily in lighter compounds. By considering an amorphous oligomer film as low-molar-mass limit of the polymer case, we expect that oligomers can flow under force even at room temperature on timescales ranging between minutes and a few hours, thus suffering irreversible deformations. A process duration considerably larger than high- $T$ embossing provides ample time for the oligomer to move and conform with the mold. Moreover, the amorphous character of the employed films, preserved by lithography, favors the patterning process, as eventual crystallites usually suppress the flow of the material. ${ }^{[15]}$ Similarly to conventional NIL, the imprinting stops when the target material has a greater contact area with the features of the stamp, as this decreases the effective pressure on the film.

Finally, we believe that an important role in RT-NIL is also played by the contraction of the free volume, $v_{\mathrm{f}}$, in the oligomer film upon pressing, which can be defined as: $v_{\mathrm{f}}=v-v_{0}$, where $v$ is the specific molecular volume, and $v_{0}$ is the characteristic incompressible volume (hard core) of the molecule. $v_{\mathrm{f}}$ is increased by conformational states preventing a dense molecular packing, which are populated at room temperature in the amorphous film, and favored by the steric hindrance of the substituent groups of our compounds. The free volume has been estimated to be up to $30 \%$ of the overall volume of macromolecule films. ${ }^{[8]}$ Its contraction can favor the deformation of the profile of the organic film during the imprinting.

We could not see any significant effect of the eventual residual solvent in the spin-cast samples, as RT-NIL was successful with amorphous films of different ages, whose solvent component is expected to be different.

In our experiments, we found that the applied pressure is the key parameter in defining the printing quality and in avoiding the detachment of the organic film from the substrate. Molecules of lower molecular weight (1-4 in Table 1) generally require less pressure in order to be imprinted without loss of adhesion with glass. Long pressing times (a few hours) can also be applied to achieve deeper imprinted patterns. So far, we have been able to produce oligomer features with an aspect ratio of up to 1 . 
Table 1. Molecular weights, applied forces during RT-NIL, emission and absorption spectral properties, and PL quantum yields of the imprinted compounds, before and after the lithography process.

\begin{tabular}{ccccccccccccc}
\hline & \multicolumn{11}{c}{ Before RT-NIL } & \multicolumn{10}{c}{ After RT-NIL } \\
\hline Compound & $\mathrm{MW}$ & $\begin{array}{c}F \\
{[\mathrm{kN}]}\end{array}$ & $\begin{array}{c}\lambda_{\mathrm{PL}} \\
{[\mathrm{nm}]}\end{array}$ & $\begin{array}{c}\Delta \lambda_{\mathrm{PL}} \\
{[\mathrm{nm}]}\end{array}$ & $\begin{array}{c}\lambda_{\text {Abs }} \\
{[\mathrm{nm}]}\end{array}$ & $\begin{array}{c}\Delta \lambda_{\text {Abs }} \\
{[\mathrm{nm}]}\end{array}$ & $\begin{array}{c}\eta_{\mathrm{PL}} \\
{[\%]}\end{array}$ & $\begin{array}{c}\lambda_{\mathrm{PL}} \\
{[\mathrm{nm}]}\end{array}$ & $\begin{array}{c}\Delta \lambda_{\mathrm{PL}} \\
{[\mathrm{nm}]}\end{array}$ & $\begin{array}{c}\lambda_{\text {Abs }} \\
{[\mathrm{nm}]}\end{array}$ & $\begin{array}{c}\Delta \lambda_{\text {Abs }} \\
{[\mathrm{nm}]}\end{array}$ & $\begin{array}{c}\eta_{\mathrm{PL}} \\
{[\%]}\end{array}$ \\
\hline $\mathbf{1}$ & 408 & 3 & 534 & 78 & 405 & 97 & 9.6 & 530 & 69 & 400 & 129 & 17.1 \\
$\mathbf{2}$ & 516 & 3 & 419 & 35 & 357 & 119 & 17.6 & 418 & 48 & 355 & 121 & 20.4 \\
$\mathbf{3}$ & 584 & 3 & 628 & 91 & 489 & 133 & 2.7 & 626 & 87 & 473 & 175 & 6.0 \\
$\mathbf{4}$ & 588 & 3 & 505 & 79 & 380 & 143 & 34.1 & 501 & 78 & 395 & 126 & 58.6 \\
$\mathbf{5}$ & 668 & 3.5 & 594 & 90 & 445 & 148 & 12.0 & 591 & 88 & 451 & 162 & 13.3 \\
$\mathbf{6}$ & 822 & 4.5 & 567 & 103 & 406 & 121 & 15.3 & 567 & 104 & 407 & 98 & 18.6 \\
$\mathbf{7}$ & 940 & 5 & 586 & 97 & 449 & 130 & 18.0 & 586 & 96 & 453 & 135 & 22.0 \\
\hline
\end{tabular}

The increase of $\eta_{\mathrm{PL}}$ in organic films after patterning can be attributed to variations of the angular distribution of the light emission, induced by the periodic surface modulation. ${ }^{[21]}$ In the untextured films, the higher refractive index with respect to the surrounding layers (i.e., glass and air), determines an enhanced waveguiding effect in the slab, and a consequent reduction of the external luminescence efficiency because of the long paths involved in selfabsorption. The imprinting of a surface periodic grating with wavelength-scale periodicity enhances the light emitted

We underline that RT-NIL is particularly suitable for lightemitting molecules. In contrast to the case of inert polymers, NIL of active materials has to be carried out under vacuum, ${ }^{[16]}$ in order to avoid the degradation of their optical and electrical properties, which are mainly induced by oxygen incorporation and substitution during the high-temperature imprinting process. ${ }^{[17]}$ Vacuum or nitrogen environments, which have been used to prevent luminescence quenching in light-emitting molecules, ${ }^{[16]}$ usually require quite a complex set-up, such as large vacuum chambers with controlled atmospheres surrounding the NIL press. To overcome this problem, other room-temperature processes have been proposed for conductive polymers, including simultaneous pressing and UV-curing $^{[18]}$ and micromolding in capillaries. ${ }^{[19]}$ In this framework, RT-NIL offers the appealing advantage that patterning can be entirely carried out in air, with no need for complex vacuum set-up. We underline that RT-NIL could be applied to many different small molecules, in addition to those employed in our study, such as tris(8-hydroxyquinoline) aluminum ( $\left.\mathrm{Alq}_{3}\right)$, biphenyl-butylphenyl-oxadiazoles (PBD), and rare-earthbased metallo-organic complexes, which are widely used for organic-based light-emitting devices and lasers. ${ }^{\text {[20] }}$

In order to assess the possible influence of RT-NIL on the optical properties of low-molar-mass organic semiconductors, we collected the Abs and PL spectra before and after the lithography process (Fig. 5). For all the molecules used, the integrated PL spectra show variation in the emission peak wavelength, $\lambda_{\mathrm{PL}}$, of less than $0.8 \%$, whereas the Abs spectra exhibit a shift up to about $4 \%$ in the peak wavelength, $\lambda_{\mathrm{Abs}}$, and a linewidth (full width at half maximum, FWHM) change up to $33 \%$ (Table 1). Further experimental work is currently in progress in our laboratory to assess the origin of the observed variations in the spectra after RT-NIL, which could be related to different packing geometries induced by the applied pressure.

For all the investigated films, we found that after patterning with periodic gratings, the external PL quantum efficiency exhibits a clear increase by a factor between 1.1 and 2.2. This indicates that no degradation of the luminescence properties of the active materials occurs after the RT-NIL, even if the process is not performed in a controlled atmosphere. towards certain forward directions and reduces the effective length covered by the photons and the self-absorption inside the organic slab. In linear distributed feedback microcavities, ${ }^{[22]}$ the angle of the emission direction, $\Theta$, is governed by the conservation of momentum in the plane of the waveguide:

$\frac{2 \pi \sin \Theta}{\lambda}= \pm \frac{2 \pi n_{\mathrm{eff}}}{\lambda} \pm m \frac{2 \pi}{\Lambda}$

where $\lambda$ is the wavelength, $n_{\text {eff }}$ is the effective refractive index of the waveguide mode, $\Lambda$ is the period of the nanostructure, and $m$ is an integer indicating the diffraction order inside the slab. For instance, with our compounds and $\Lambda=600 \mathrm{~nm}$, Equation 1 leads to emission angles between about $35^{\circ}$ and $70^{\circ}$ for the first-order Bragg scattering. This confirms the enhancement of the forward output. In addition we note that, notwithstanding the well preserved integrated emission spectra, a few nanometers blue-shift of $\lambda_{\mathrm{PL}}$ is often observed after patterning (Table 1), which suggests a reduced self-absorption, which is due to the overlap between emission and absorption in the short-wavelength tail of the PL. A deeper understanding of the angular distribution of the emitted light is obtained by measuring the PL emission at different angles, $\Theta$. The forward PL (measured for light emitted in a small

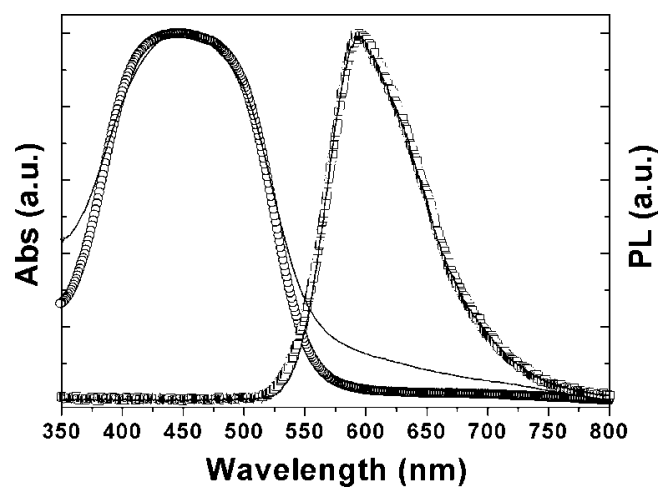

Figure 5. Abs and integrated PL spectra before (empty circles and squares, respectively) and after (superimposed lines) RT-NIL for a prototype low-molar-mass light-emitting molecule (5). Similar results hold for all the other investigated molecules. 
solid angle $\sim 10^{-3} \mathrm{rad}$ around $\Theta=38^{\circ[23]}$ ) from a patterned oligomer film (6), is compared to the spectrum of the untextured sample in Figure 6. The enhanced emission peak of the printed distributed feedback microcavity is clearly visible at

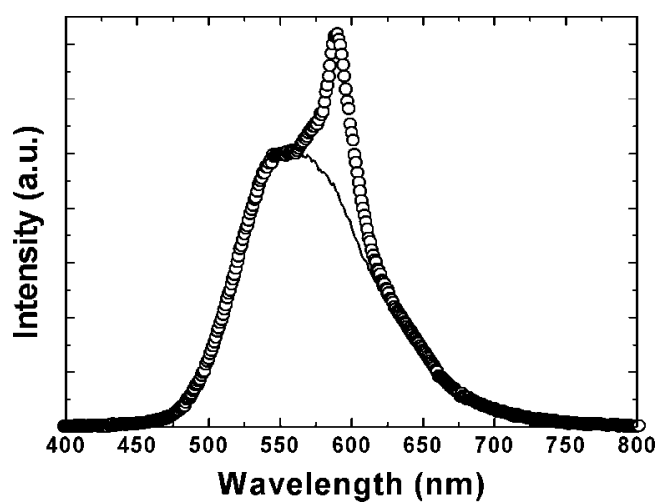

Figure 6. $\mathrm{PL}$ spectra at an emission polar angle, $\Theta=38^{\circ}$, for a $600 \mathrm{~nm}$ period patterned (line) and an un-patterned (empty dots) film of the molecule 6

$590 \mathrm{~nm}$. Similar results were measured in all the patterned samples. The diffraction peak wavelength depends on the collection angle, as the detection is moved perpendicularly to the direction of the patterned grooves, according to a guided Bragg mode (Eq. 1), in agreement with previously reported experiments onto patterned organic slabs. ${ }^{[21,22]}$

These finding clearly demonstrate that RT-NIL is a useful and easy technique to transfer patterns with $100 \mathrm{~nm}$ scale resolution on to low-molar-mass organic semiconductors, which are difficult to print otherwise using conventional embossing. The output optical mode density of light-emitting films is thus effectively controlled. This substantially extends the solidstate patterning technologies to novel compounds, resulting in important advances towards fully plastic electronics and photonics.

Received: August 12, 2003 Final version: October 13, 2003

[1] a) S. Y. Chou, P. R. Krauss, P. J. Renstrom, Appl. Phys. Lett. 1995, 67, 3114. b) S. Y. Chou, P. R. Krauss, P. J. Renstrom, Science 1996 , 272,85 .

[2] B. Heidari, I. Maximov, L. Montelius, J. Vac. Sci. Technol. B 2000, $18,3557$.

[3] S. Y. Chou, P. R. Krauss, W. Zhang, L. Guo, L. Zhuang, J. Vac. Sci. Technol. B 1997, 15, 2897.

[4] L. Guo, P. R. Krauss, S. Y. Chou, Appl. Phys. Lett. 1997, 71, 1881

[5] Z. Yu, S. J. Schablitsky, S. Y. Chou, Appl. Phys. Lett. 1999, 74, 2381.

[6] M. Li, L. Chen, S. Y. Chou, Appl. Phys. Lett. 2001, 78, 3322.

[7] D. Pisignano, M. Anni, G. Gigli, R. Cingolani, M. Zavelani-Rossi, G. Lanzani, G. Barbarella, L. Favaretto, Appl. Phys. Lett. 2002, 81, 3534.

[8] D.-Y. Khang, H. Yoon, H. H. Lee, Adv. Mater. 2001, 13, 749.

[9] G. Barbarella, L. Favaretto, G. Sotgiu, L. Antolini, G. Gigli, R. Cingolani, A. Bongini, Chem. Mater. 2001, 13, 4112.
[10] G. Barbarella, L. Favaretto, G. Sotgiu, M. Zambianchi, V. Fattori, M. Cocchi, F, Cacialli, G. Gigli, R. Cingolani, Adv. Mater. 1999, 11, 1375.

[11] G. Barbarella, L. Favaretto, G. Sotgiu, M. Zambianchi, A. Bongini, C. Arbizzani, M. Mastragostino, M. Anni, G. Gigli, R. Cingolani, J. Am Chem. Soc. 2000, 122, 11971.

[12] K. Y. Suh, Y. S. Kim, H. H. Lee, Adv. Mater. 2001, 13, 1386.

[13] S. Zankovych, T. Hoffmann, J. Seekamp, J.-U. Bruch, C. M. Sotomayor Torres, Nanotechnology 2001, 12, 91.

[14] Physical Properties of Polymers Handbook (Ed: J. E. Mark), American Institute of Physics Press, Woodbury, NY 1996.

[15] G. Strobl, The Physics of Polymers, Springer, Berlin 1997.

[16] J. Wang, X. Sun, L. Chen, S. Y. Chou, Appl. Phys. Lett. 1999, 75 , 2767.

[17] a) M. Yan, L. J. Rothberg, F. Papadimitrakopoulos, M. E. Galvin, T. M. Miller, Phys. Rev. Lett. 1994, 73, 744. b) F. Papadimitrakopoulos, K. Konstadinidis, T. M. Miller, R. Opila, E. A. Chandross, M. E. Galvin, Chem. Mater. 1994, 6, 1563.

[18] M. Behl, J. Seekamp, S. Zankovych, C. M. Sotomayor Torres, R. Zentel, J. Ahopelto, Adv. Mater. 2002, 14, 588.

[19] W. S. Beh, I. T. Kim, D. Qin, Y. Xia, G. M. Whitesides, Adv. Mater. 1999, 11, 1038.

[20] a) V. G. Kozlov, V. Bulovic, P. E. Burrows, S. R. Forrest, Nature 1997, 389, 362. b) A. Köhler, J. S. Wilson, R. H. Friend, Adv. Mater 2002, 14, 701 .

[21] B. J. Matterson, J. M. Lupton, A. F. Safonov, M. G. Salt, W. L. Barnes, I. D. W. Samuel, Adv. Mater. 2001, 13, 123.

[22] G. A. Turnbull, P. Andrews, M. J. Jory, W. L. Barnes, I. D. W. Samuel, Phys. Rev. B: Condens. Matter 2001, 64, 125122.

[23] For a given wavelength, the waveguided mode couples to free radiation only for a very small angular range around $\Theta$, in agreement with Equation 1. For this reason the Bragg peaks are not visible in the angle-integrated PL spectrum shown in Figure 4.

\section{From Hollow to Dense Spheres: Control of Dipolar Interactions by Tailoring the Architecture in Colloidal Aggregates of Superparamagnetic Iron Oxide Nanocrystals $* *$}

\author{
By Pedro Tartaj,* Teresita González-Carreño, \\ and Carlos J. Serna
}

The fabrication of colloids with desired properties has been the aim of many recent investigations. The modification of colloids was found to be a powerful tool for drastically changing or precisely tuning their optical, mechanical, and surface properties. ${ }^{[1]}$ Of special interest for applications in biotechnology and for studying fundamental phenomena in physics is

[*] Dr. P. Tartaj, Dr. T. González-Carreño, Prof. C. J. Serna Instituto de Ciencia de Materiales de Madrid, CSIC Cantoblanco, E-28049 Madrid (Spain) E-mail: ptartaj@icmm.csic.es

[***] Financial support from CICYT (MAT2002-04001-C02) is gratefully acknowledged. P. Tartaj thanks the financial support from the Ramon y Cajal program. 